IC/92/196, SISSA-140/92/EP, LMU-09/92

August 1992

\title{
PLANCK SCALE EFFECTS IN NEUTRINO PHYSICS
}

\author{
Eugeni Kh. Akhmedov ${ }^{(a, b, c)}$ đ, Zurab G. Berezhiani ${ }^{(d, e)}$ 中田, \\ Goran Senjanović( $^{(a)}$ हf, Zhijian Tao ${ }^{(a)}$ \\ ${ }^{(a)}$ International Centre for Theoretical Physics, I-34100 Trieste, Italy \\ (b) Scuola Internazionale Superiore di Studi Avanzati, I-34014 Trieste, Italy \\ (c) Kurchatov Institute of Atomic Energy, Moscow 123182, Russia \\ (d) Sektion Physik der Universität München, D-8000 Munich-2, Germany \\ (e) Institute of Physics, Georgian Academy of Sciences, Tbilisi 38007r, Georgia
}

\begin{abstract}
We study the phenomenology and cosmology of the Majoron (flavon) models of three active and one inert neutrino paying special attention to the possible (almost) conserved generalization of the Zeldovich-Konopinski-Mahmoud lepton charge. Using Planck scale physics effects which provide the breaking of the lepton charge, we show how in this picture one can incorporate the solutions to some of the central issues in neutrino physics such as the solar and atmospheric neutrino puzzles, dark matter and a $17 \mathrm{keV}$ neutrino. These gravitational effects induce tiny Majorana mass terms for neutrinos and considerable masses for flavons. The cosmological demand for the sufficiently fast decay of flavons implies a lower limit on the electron neutrino mass in the range of $0.1-1 \mathrm{eV}$.
\end{abstract}

\footnotetext{
*E-mail: akhmedov@tsmi19.sissa.it, akhm@jbivn.kiae.su

${ }^{\dagger}$ Alexander von Humboldt Fellow

${ }^{\ddagger}$ E-mail: zurab@hep.physik.uni-muenchen.de, vaxfe::berezhiani

§E-mail: goran@itsictp.bitnet, vxicp1::gorans
} 


\section{Introduction}

The central open issues in neutrino physics, according to our belief, are

(a) The solar neutrino puzzle (SNP). The solar neutrino experiments under operation [1, 2, 3, 4] indicate a deficiency of solar neutrinos pointing to neutrino properties being a source of the discrepancy between theory and experiment. The most popular and natural explanation is based on oscillations of $\nu_{e}$ into another neutrino in solar matter or in vacuum during the flight to the earth.

(b) The atmospheric neutrino puzzle (ANP). There is some evidence for a significant depletion of the atmospheric $\nu_{\mu}$ flux, by almost a factor of two [5]. This result, if true, would point again to neutrino oscillations, this time of $\nu_{\mu}$ into another species, with a large mixing angle and an oscillation length less than or of the order of the atmospheric height.

It is, at least in principle, possible to resolve both the SNP and ANP in the context of the usual three neutrino flavors, e.g. the SNP could be due to the $\nu_{e} \rightarrow \nu_{\mu}$ oscillations, and the ANP due to the $\nu_{\mu} \rightarrow \nu_{\tau}$ oscillations.

(c) Dark matter problem. Neutrinos with a mass in the range of 10-100 eV have been considered for many years as natural candidates for dark matter needed to explain the observed large scale structure of the universe. This popular, so-called hot dark matter (HDM) scenario, also able to explain the missing cosmological density, was disfavored in the last years due to the bounds on the primordial density fluctuations coming from the measurements of the cosmic microwave background radiation (CMBR). The recent COBE discovery of the CMBR anisotropy [6], however, suggests at least some presence of HDM together with cold dark matter (CDM) with the latter being the dominant component [7]. This role can now be naturally played by neutrinos with a mass in eV range.

(d) A $17 \mathrm{keV}$ neutrino. As exciting as it is, the existence of the $17 \mathrm{keV}$ neutrino is far from being established [8, 9]. Many theoretical models on the subject were proposed 
[10], but the difficult task of incorporating the SNP in this picture has only recently been addressed [11]. The problem is that the conventional scenario of three neutrinos $\nu_{e}, \nu_{\mu}$ and $\nu_{\tau}$ cannot reconcile laboratory constraints with solar neutrino deficit. Namely, the combined restriction from the neutrinoless double $\beta$ decay and $\nu_{e} \leftrightarrow \nu_{\mu}$ oscillations leads to a conserved (or at most very weakly broken) generalization of ZKM [12] symmetry: $L_{e}-L_{\mu}+L_{\tau}$ [13]. This in turn implies the $17 \mathrm{keV}$ neutrino $\nu_{17}$ mainly to consist of $\nu_{\mu}^{c}$ and $\nu_{\tau}$, mixed by the Simpson angle $\theta_{S} \simeq 0.1$ with the massless $\nu_{e}$. Clearly, in this picture there is no room for the solution of the SNP due to neutrino properties.

It is well-known by now that the LEP limit [14] on $Z^{0}$ decay width excludes the existence of yet another light active neutrino. However, the same in general is not true for a sterile neutrino $\left(n_{R}\right)$. Of course, once introduced, $n$ (instead of $\left.\nu_{\mu}^{c}\right)$ can combine with the $\nu_{\tau}$ to form $\nu_{17}$ or just provide a missing light partner to $\nu_{e}$ needed for the neutrino oscillation solution to the SNP. The latter possibility has been recently advocated by the authors of ref. [11]. In this paper we study in some detail the physics of an extra sterile neutrino. We will show that its existence can accommodate the solution to all the above puzzles. We offer a systematic study of this scenario, paying special attention to possible effective operators that could induce neutrino masses. We consider the case of a maximal abelian lepton flavor symmetry with $n_{R}$ included, inspired by an analysis performed by Barbieri and Hall (BH) [15] for the case of three active neutrinos. The crucial characteristics of this approach is the existence of flavons, i.e. Majorons associated with spontaneous violation of extended lepton flavor symmetries. These flavons can naturally provide sufficiently fast decay of $\nu_{17}$ which is necessary for cosmological reasons.

In order to generate neutrino oscillations in the light sector needed for the solution of the SNP and ANP, it will turn out necessary to break the lepton number symmetry. We propose an interesting possibility of higher dimensional operators being responsible for this breaking [16, 17]. These operators could naturally result from the quantum gravitational effects and should be cut off by the Planck scale. We find it encouraging that such tiny 
effects may be sufficient for the simultaneous solution of the above mentioned problems. It will be shown in section 3 that these effects induce mass splittings between the components of Dirac or ZKM neutrinos of the order of $10^{-6} \mathrm{eV}$. Since the solution to ANP seems to require $\Delta m^{2} \simeq 10^{-2}-10^{-3} \mathrm{eV}^{2}$ with large mixing angles, this in turn suggests that the mass of the heavy neutrino is of the order of a few $\mathrm{keV}$. This encouraged us to seriously pursue the possibility of a controversial $17 \mathrm{keV}$ neutrino, although all we need is the existence of a heavy neutrino with a mixing angle which could be much smaller than $\theta_{S}$. We would like to emphasize that otherwise our analysis is quite general, and it will hold true even if $\nu_{17}$ with $\theta_{S} \simeq 0.1$ disappears problem by inducing appreciable masses for flavons, of the order of $1 \mathrm{keV}$. Just like $\nu_{17}$, they also must decay fast enough in order not to postpone the matter dominated era of the expansion of the universe needed for the development of the cosmological large scale structure. This requirement is put on the firmer ground through the COBE findings indicating rather small initial density fluctuations. Since the couplings of Majorons to neutrinos are necessarily proportional to the masses of the latter, this leads to both phenomenologically and cosmologically important lower limit on the electron neutrino mass $m_{\nu_{e}}>(0.1-1) \mathrm{eV}$. As we will show in the section 3, electron neutrino thus becomes a natural candidate to provide the needed 10-30 per cent hot dark matter of the universe.

Our paper is organized as follows. In the next section we offer a general study of a system of three active and one sterile neutrino with a conserved generalized ZKM lepton number. In the section 3 we study the implications of the necessary breaking of this symmetry induced through the quantum gravitational effects. In section 4 a specific model is offered for the sake of demonstration and finally the last section is reserved for the discussion and outlook.

\footnotetext{
${ }^{1}$ Hereafter " $\nu_{17}$ " denotes a heavy neutrino, even if its mass is not exactly $17 \mathrm{keV}$ and its mixing is different from the Simpson angle.
} 


\section{The effective operator study of the neutrino masses}

The introduction of a new state $n_{R}$ opens up a number of new possibilities for a conserved (or approximately conserved) generalized lepton number $L$. We distinguish two such different classes.

(i) A case of one Dirac and one ZKM state, for which $L$ takes the form

$$
L_{ \pm}=L_{e}-L_{\mu} \pm\left(L_{\tau}-L_{n^{c}}\right)
$$

where hereafter we use the convenient notation of a left-handed $n^{c}$ field $\left(n^{c}\right)_{L} \equiv C \bar{n}_{R}^{T}$. Notice that $L_{e}$ and $L_{\mu}$ charges must enter with opposite signs in order to comply with the absence of $\nu_{e} \leftrightarrow \nu_{\mu}$ oscillation with a Simpson mixing angle $\theta_{S} \simeq 0.1$. In each of $L_{+}$and $L_{-}$case, we are still left with the freedom of having $\nu_{17} \simeq \nu_{\tau}+n_{R}$ or $\nu_{17} \simeq \nu_{\tau}+\nu_{\mu}^{c}$ for $L_{+}$and $\nu_{17} \simeq \nu_{\mu}+n_{R}$ or $\nu_{17} \simeq \nu_{\tau}+n_{R}$ for $L_{-}$. If $n_{R}$ is a part of $\nu_{17}$, one is potentially in conflict with the supernova 1987A bound $m_{17} \leq(1-30) \mathrm{keV}$ due to $n$ being sterile and taking away the energy of the supernova after a helicity flipping scattering $\nu_{\tau}\left(\nu_{\mu}\right) \rightarrow n$ [18]. The same limit does not apply, of course, when $\nu_{17}$ consists of only active neutrinos.

(ii) A case of either Dirac or ZKM $\nu_{17}$ and two massless states, corresponding to lepton charges with only one minus sign:

$$
\begin{aligned}
& L_{1}=L_{e}-L_{\mu}+L_{\tau}+L_{n^{c}} \\
& L_{2}=L_{e}+L_{\mu}-L_{\tau}+L_{n^{c}} \\
& L_{3}=L_{e}+L_{\mu}+L_{\tau}-L_{n^{c}}
\end{aligned}
$$

Obviously, $-L_{e}$ is not allowed.

In what follows, we shall analyse systematically the above possibilities, some of which were studied in the context of specific models [19]. Our aim is to extract as much as possible model independent information, but we will also present a simple model which will serve as an illustration of general considerations. 
One may wonder at this point whether $\nu_{i} \rightarrow n$ oscillations could bring $n$ into equilibrium before the nucleosynthesis [20, 21, 22, thereby affecting the primordial ${ }^{4} \mathrm{He}$ abundance in the universe [23]. The situation crucially depends on the mixing angle $\theta_{n}$ between sterile and active neutrinos and so varies with the structure of $\nu_{17}$.

The relative presence of $n$ in the number of neutrino species at the time of nucleosynthesis is of course a function of its decoupling temperature $T_{n}$. We can thus speak of two distinct cases, $T_{n}>T_{Q C D}$ and $T_{n} \leq T_{Q C D}$, where $T_{Q C D}$ is the QCD phase transition temperature. In the former case, it can be shown that $n$ counts at most 0.3 of the usual neutrino contribution due to the reheating of active neutrinos when $T$ drops below $T_{Q C D}$, whereas in the latter case we expect $N_{\nu} \simeq 4$, since the only change below $T_{Q C D}$ is the annihilation of $\mu^{+} \mu^{-}$pairs, which barely changes the temperature of the neutrino sea. Clearly, $T_{n}$ depends on the mixing angle $\theta_{n}$, the smaller $\theta_{n}$ is, the larger $T_{n}$.

From an analysis of ref. [20] one can get (for a mass difference $\Delta m \simeq 17 \mathrm{keV}$ ) the relation between $\theta_{n}$ and $T_{n}$ :

$$
T_{n}^{3} \simeq \frac{(3 \mathrm{MeV})^{3}}{1 / 2 \sin ^{2} 2 \theta_{n}}
$$

and $T_{n} \geq T_{Q C D} \simeq 200 \mathrm{MeV}$ requires $\theta_{n} \leq 10^{-3}$.

Now it is readily seen that for $L_{-}$the mixing angle $\theta_{n}$ coincides with $\theta_{S}$ and therefore in this case one predicts $N_{\nu} \simeq 4$ ( since $T_{n} \ll T_{Q C D}$ ). In other cases the situation depends on the details of the model, i.e. on the structure of $\nu_{17}$; we will return to them later when we discuss the neutrino mass matrix.

Before proceeding, we wish to recall the fact that $\nu_{17}$ must decay fast enough in order to comply with cosmological constraints, and it appears that the simplest mechanism is provided by the Majoron, the Goldstone boson of a spontaneously broken lepton number (or lepton flavor) symmetry. We therefore assume large global symmetry $G$ spontaneously broken down to $L$. In particular, this implies the existence of some new scalar fields, generically denoted by $S$, which transform nontrivially under $G$. Since the Majoron (one 
or more) is a phase of $S$, due to already mentioned LEP constraints on the $Z^{0}$ decay width $S$ fields should be singlets under $S U(2)_{L} \times U(1)$. Furthermore, any effective mass term invariant under $G$ will necessarily involve $S$ fields (assuming that the lepton flavor numbers, including $n$, are distinct). When an illustration is needed, we discuss the straightforward extension of lepton number based on $G=U(1)_{e} \times U(1)_{\mu} \times U(1)_{\tau} \times U(1)_{n}$.

In order to generate small masses naturally, we allow no tree-level $d=4$ operators that could lead to neutrino masses. In particular, this implies that

(a) the only scalar fields are $S U(2)_{L}$ doublets and singlets, and

(b) no singlet carries such quantum numbers under $G$ as to give direct $(d=4)$ Yukawa couplings.

In the context of the above example we allow only $S_{a b}, a \neq b(a, b=e, \mu, \tau, n)$ singlet fields. These fields give naturally rise to "flavons", i.e. Majorons which change lepton flavor and provide fast decay of the $\nu_{17}$ [15].

Consistent with the above rules, the leading effective Yukawa neutrino operators invariant under $S U(2)_{L} \times U(1) \times G$ are

$$
\alpha_{i j}\left(l_{i}^{T} C \tau_{2} \vec{\tau} l_{j}\right) \frac{H^{T} \tau_{2} \vec{\tau} H}{M^{2}} S_{i j}^{*}, \quad \alpha_{i n}\left(l_{i}^{T} C n^{c}\right) \frac{\tau_{2} H S_{i j}^{*} S_{j n}}{M^{2}}
$$

where $l_{i}^{T}=\left(\nu_{i L}^{T}, e_{i L}^{T}\right)$ are the leptonic weak doublets, $H$ is the usual $S U(2)_{L} \times U(1)$ Higgs doublet, $M$ is a regulator (cut-off) scale which is an input parameter and should be above $<H>\sim M_{W}$ and $\left\langle S>\left(S\right.\right.$ generically denotes $S_{i j}$ and $\left.S_{i n}\right)$, and $\alpha_{a b}$ are dimensionless factors expected to arise from the loop expansion, $\alpha_{a b} \leq 10^{-3}-10^{-4}$. The quantum numbers of $S_{a b}$ fields under $G=U(1)_{e} \times U(1)_{\mu} \times U(1)_{\tau} \times U(1)_{n}$ are

$$
\begin{array}{ll}
S_{e \mu}(1,1,0,0) & S_{e n}(1,0,0,1) \\
S_{\mu \tau}(0,1,1,0) & S_{\mu n}(0,1,0,1) \\
S_{e \tau}(1,0,1,0) & S_{\tau n}(0,0,1,1)
\end{array}
$$

Leptons carry their usual flavor charges, $n^{c}$ carries -1 unit of $U(1)_{n}$, and the Higgs doublet 
$H$ of course carries no lepton flavor. From the constraints on $\nu_{17}$ flavon decay, one can deduce the limit $30 \mathrm{GeV} \leq<S>\leq 300 \mathrm{GeV}$ [15], where the non-vanishing $<S>$ conserve lepton number $L$ (for any $L$ defined above there corresponds a certain set of $<S>$ ).

Before one specifies the form of $\nu_{17}$ in the sense discussed above, one cannot decide the value of $M$ and $\left\langle S>\right.$. For example, if $\nu_{17} \simeq \nu_{\tau}+n$, both $\langle S>$ and $M$ could be as large as desired, whereas in the case $\nu_{17} \simeq \nu_{\tau}+\nu_{\mu}^{c}$ both $M$ and $\langle S\rangle$ should be close to $M_{W}$ (see eq. (21) below). We come back to this question in the specific examples, suffice is to say that the operators (4) give the leading contributions to neutrino masses. We start for definiteness with $L_{+}=L_{e}-L_{\mu}+L_{\tau}-L_{n^{c}}$, in which case the non-vanishing VEVs are $<S_{e \mu}>,<S_{\mu \tau}>$ and $<S_{\mu n}>$. The neutrino mass matrix in the Dirac basis takes then the form

$$
M_{\nu}=\begin{array}{cc}
n^{c} & \nu_{\mu} \\
\nu_{\tau}
\end{array}\left(\begin{array}{cc}
m_{e n} & m_{e \mu} \\
m_{\tau n} & m_{\tau \mu}
\end{array}\right)
$$

From the smallness of the Simpson's angle and the absence of the $\nu_{\mu} \rightarrow X$ oscillations it follows that only one entry of $M_{\nu}$, either $m_{\tau n}$ or $m_{\tau \mu}$, can be $\sim 17 \mathrm{keV}$, whereas the other entries must be at least an order of magnitude smaller. As we mentioned before, there is still freedom for $\nu_{17}$ to consist of either (a) $\nu_{\tau}+n$ or (b) $\nu_{\tau}+\nu_{\mu}^{c}$, depending on whether $m_{\tau n}$ or $m_{\tau \mu}$ is large, respectively. In the former case, $\theta_{S} \simeq m_{e n} / m_{\tau n}$ while in the latter, $\theta_{S} \simeq m_{e \mu} / m_{\tau \mu}$. The angle $\theta_{n} \simeq m_{\tau \mu} / m_{\tau n}$ (a) or $\theta_{n} \simeq m_{\tau n} / m_{\tau \mu}$ (b) determines the abundance of $n$ during the nucleosynthesis. If it is less than $10^{-3}$, we expect $N_{\nu} \leq 3.3$ and, if not, $N_{\nu}$ is close to 4 .

The analysis for other choices of $L$ can easily be performed along the same lines and we do not present it here. 


\section{The only good global symmetry is a broken global symmetry}

As we have seen up to now, in the limit of exact $L$ the neutrino spectrum prevents oscillations in the light sector and so leaves the SNP unresolved. On the other hand, the belief in exact global symmetries is becoming increasingly less popular. This is certainly encouraged by the fact that the virtual black holes and wormholes, while preserving local gauge invariance, can destroy the meaning of global quantum numbers. It is not unlikely then that there could be higher dimensional operators cut off by the Planck scale which violate our lepton number symmetry. Barring the possibility of accidental cancellations and assuming that the symmetry $G$ is not a part of a larger local gauge symmetry, we expect this breaking to occur at the $d=5$ effective operator level.

Neutrino mass. Without further ado then, we list the leading operators that could induce corrections to the neutrino mass matrix [16, 17]:

$$
\left(\nu_{i}^{T} C \tau_{2} \vec{\tau} \nu_{i}\right) \frac{H^{T} \tau_{2} \vec{\tau} H}{M_{\mathrm{Pl}}}, \quad\left(n^{T} C n\right)\left[\frac{H^{\dagger} H}{M_{\mathrm{Pl}}}+\frac{S^{2}}{M_{\mathrm{Pl}}}\right]
$$

where $S^{2}$ stands for any bilinear combinations of the $S_{a b}$ fields, and we list only the flavordiagonal terms since their effect on $M_{\nu}$ is most dramatic. Namely, they induce the mass splittings between the components of Dirac and ZKM neutrinos and open up new channels for oscillations.

The split $\Delta m$ coming through the above operators when the relevant fields get nonvanishing VEVs can be estimated as

$$
\Delta m \leq \frac{M_{W}^{2}}{M_{\mathrm{Pl}}} \simeq 10^{-6} \mathrm{eV}
$$

where the number $10^{-6} \mathrm{eV}$ is probably an upper limit, since there could be further dimensionless suppressions in (8) (certainly an order of magnitude suppression should not come out as a surprise). We remind the reader that our mass scales are expected to lie close to 
the electroweak scale. An important point is that the gravitational effects are expected to be flavor blind. This implies that the mass splits in the light and heavy sectors should be of the same order of magnitude. These splits $\Delta m$ being small, much less than any Dirac mass term, leads to the prediction of two pseudo-Dirac states with the mixing between the partners in each state being maximal $\left(\simeq 45^{\circ}\right)$.

The squared mass differences in the light and heavy sectors will be

$$
\Delta m_{\text {light }}^{2} \simeq m_{\nu_{e}} \Delta m, \quad \Delta m_{\text {heavy }}^{2} \simeq m_{17} \Delta m
$$

where $\Delta m$ is given by eq. (8) and $m_{17}$ is the mass of heavy neutrino. The experimental upper limit is $m_{\nu_{e}}<9.4 \mathrm{eV}$ [24] and, as we shall see below from the discussion of the Majoron decays, there is a lower limit $m_{\nu_{e}}>0.1 \mathrm{eV}$ implying $10^{-8} \mathrm{eV}^{2}<\Delta m_{\text {light }}^{2}<10^{-5}$ $\mathrm{eV}^{2}$. This range allows for the neutrino oscillations being naturally the solution of the SNP.

The oscillations in the heavy sector $\nu_{\mu} \rightarrow \nu_{\tau}\left(n^{c}\right)$ can be relevant for the recently reported deficiency of the atmospheric $\nu_{\mu}$ [5]. From eq. (9) it follows that for $m_{17} \sim 10 \mathrm{keV} \Delta m_{\text {heavy }}^{2}$ can naturally be $\sim 10^{-2}-10^{-3} \mathrm{eV}^{2}$ which with the mixing angle being $45^{\circ}$ perfectly fits the required parameter range [5].

The induced mass splittings of the pseudo-Dirac neutrinos open up new channels of oscillations that can bring the sterile neutrino $n$ into the equilibrium at the time of nucleosynthesis. Although the number of allowed light species at that epoch is still debated [25], the frequently quoted limit $N_{\nu}<3.4$ [23], if taken seriously, would imply $\Delta m_{\text {light }}^{2}<5 \times 10^{-9}$ $\mathrm{eV}^{2}$ if $n$ is a part of $\nu_{\text {light }}$ and $\Delta m_{\text {heavy }}^{2}<8 \times 10^{-6} \mathrm{eV}^{2}$ when $n$ is a component of $\nu_{17}$ [22]. From the limit $\Delta m_{\text {light }}^{2}>10^{-8} \mathrm{eV}^{2}$ it is clear that we are dangerously close to the prediction of 4 light neutrino species in equilibrium, i.e. $N_{\nu}=4$. However, due to the uncertainties in the estimation of the gravitational effects, any conclusion would be premature; all we can say is that $N_{\nu}$ could be lying anywhere between 3 and 4 .

Majoron mass. As much as in the case of the neutrinos, we expect $d=5$ effective 
operators explicitly violating lepton number in the scalar sector

$$
\frac{S}{M_{\mathrm{Pl}}}\left[S^{4}+S^{2} H^{\dagger} H+\left(H^{\dagger} H\right)^{2}\right]
$$

where we only give a typical example. Therefore the Majorons (in our case flavons) acquire non-vanishing masses, i.e. become pseudo-Goldstone bosons. Since we take $<S>\sim M_{W}$, we get an order of magnitude estimate

$$
m_{F} \simeq\left(\frac{M_{W}^{3}}{M_{\mathrm{Pl}}}\right)^{1 / 2} \simeq 1 \mathrm{keV}
$$

The above is the typical value of the elements of the mass matrix of flavons $F_{a b}$ which are expected to have large mixings with each other.

Since $m_{F} \ll m_{17}$, the decay rate of $\nu_{17}$ is almost unaffected by the generated flavon masses. However, the issue now becomes whether flavons themselves manage to decay fast enough to be in accord with cosmological limits. Let us recall here the estimate of the $\nu_{17}$ lifetime due to the decay $\nu_{17} \rightarrow \nu_{e}+F$ :

$$
\tau_{17} \simeq 16 \pi\left(\theta_{S} \frac{m_{17}}{<S>}\right)^{-2} m_{17}^{-1} \simeq 10^{-1} \mathrm{~s}
$$

for $\langle S\rangle \simeq M_{W}$, which is obviously cosmologically acceptable. We should stress that $\nu_{17}$ is relativistic at the cosmological time $t \simeq(0.1-1)$ sec and so the time dilation effect makes the actual decay time in the comoving reference frame bigger than 1 sec. Therefore, flavons appear only after the nucleosynthesis.2]

However, the cosmological problems related to the $\nu_{17}$ get now replaced by the presence of massive flavons which are produced in the $\nu_{17}$ decay with the concentration being equal to that of the active neutrinos. The only possible mechanism to solve the problem of

\footnotetext{
${ }^{2}$ It is easy to see that even if flavons were in equilibrium at $T \sim<S>\sim 100 \mathrm{GeV}$ for whatever reason (e.g. due to the coupling to charged physical scalars in certain models such as the one discussed in section 4), the weakness of their interactions with neutrinos makes them decouple much before the QCD phase transition. This means that they do not count at the time of nucleosynthesis or, better to say, contribute a few percent of the usual neutrino species.
} 
an overabundance of massive flavons is their decay into light neutrinos $\nu_{e}$. Recall that the coupling of Majorons to neutrinos is proportional to the neutrino mass and this decay cannot take place for massless $\nu_{e}$. This poses a serious problem for any Majoron-type models of the $17 \mathrm{keV}$ neutrino in which $\nu_{e}$ stays massless or very light (e.g. for $L=L_{1,2,3}$, eq. (2) or in the absence of sterile neutrinos for $\left.L=L_{e}-L_{\mu}+L_{\tau}\right)$. This question was recently raised by Grasso et al. [17] in the context of the BH picture. However, in our case all we know is that $m_{\nu_{e}}<10 \mathrm{eV}$ [24] and so flavons are free to decay into light neutrinos. As we show now, this provides a lower limit on the $\nu_{e}$ mass $s^{3}$. It is easy to estimate the flavon lifetime due to the decay into two light neutrinos:

$$
\tau_{F} \simeq 8 \pi\left(\frac{m_{\nu_{e}}}{<S>}\right)^{-2} m_{F}^{-1}
$$

which using eq. (11) becomes

$$
\tau_{F} \simeq 8 \pi \frac{\left(<S>M_{\mathrm{Pl}}\right)^{1 / 2}}{m_{\nu_{e}}{ }^{2}} \simeq 10^{6}\left(\frac{\mathrm{eV}}{m_{\nu_{e}}}\right)^{2} \mathrm{~s}
$$

It is clear from the above result that no useful limit on $\tau_{F}$ (i.e. on $m_{\nu_{e}}$ ) emerges from the requirement that the universe is not overclosed. A much more serious constraint follows, however, from the galaxy formation constraint. The recent COBE measurements of CMBR anisotropy [6] implies the small initial density fluctuations $\delta \rho / \rho \sim 10^{-5}$. This, in turn, requires a sufficiently long matter-dominated epoch for the linear growth of $\delta \rho / \rho$ to form the observed large-scale structure of the universe. Therefore the decay products of the flavons have to be redshifted sufficiently in order not to dominate the non-relativistic matter (CDM) density at the time $t_{e q}$ of radiation dominated universe turning into a matter dominant one in the standard picture. We, therefore, demand

$$
m_{F} n_{\nu}\left(t_{e q}\right)\left(\frac{\tau_{F}}{t_{e q}}\right)^{1 / 2}<\frac{1}{2} \rho_{c r}\left(t_{e q}\right)
$$

where $n_{\nu}\left(t_{e q}\right)$ is neutrino number density at that time and $\rho_{c r}\left(t_{e q}\right)$ is the critical density at the same time. Using eq. (11) one obtains the limit $\tau_{F}<10^{7}$ s. This along with eq. (14)

\footnotetext{
${ }^{3}$ The general study of the cosmological implications of massive Majorons is presently being done [26].
} 
leads to the promised lower limit on the electron neutrino mass

$$
m_{\nu_{e}}>0.3 \mathrm{eV}
$$

where due to uncertainties in the flavon masses and mixings, this limit should be read as some number between 0.1 and $1 \mathrm{eV}$.

We should stress here that increasing the scale $<S>$ of the lepton symmetry breaking only increases the lower limit on $m_{\nu_{e}}$ since both $m_{F}$ and $\tau_{F}$ become larger. Moreover, at the scale $\langle S\rangle \gg 1 \mathrm{TeV}$ flavons become heavier than $\nu_{17}$ and therefore $\nu_{17}$ itself cannot decay.

It is rather encouraging that the limit in (16) is not too far from the laboratory upper limit on $m_{\nu_{e}}$. This provides even more impetus for the direct experimental search of electron neutrino mass in $\beta$ decays. It should be remembered that the almost Dirac nature of $\nu_{e}$ in our work implies prediction of a negative result in the experiments on neutrinoless double $\beta$ decay.

The cosmological implication of our result is also rather important. Let us notice that the concentration of light neutrinos today is eight times that of a normal two-component neutrino. Recall that before $\nu_{17}$ decay there are 4 light neutrino species, and this number will not change with just the decay of $\nu_{17}$. However, the subsequent decay of flavons adds yet another four species to the light neutrino concentration of the present day universeft. So we can estimate the present-day light neutrino concentration to be

$$
n_{\nu}=8 \times \frac{3}{11} n_{\gamma} \simeq 8701 / \mathrm{cm}^{3}
$$

where $n_{\gamma} \simeq 400 / \mathrm{cm}^{3}$ is the relic photon density of the universe. Then from (16) we find that at least a fraction of the present-day critical density of the universe is in the form of neutrinos, i.e. hot dark matter. This observation may be rather important, since the standard model of $\mathrm{CDM}$ with bias $b \simeq 2-3$ seems to be disfavored in view of recent COBE measurements, if one takes the Harrison-Zeldovich "flat" spectrum for initial density

\footnotetext{
${ }^{4}$ Each flavon decays into a pair of light neutrinos, and in decay of $\nu_{17}$ two flavons are produced and not one as it could appear at first sight. We are grateful to J. Cline for this remark.
} 
fluctuations, which is motivated (up to some small corrections) by inflation. In this case the value of the CMBR quadrupole anisotropy is more than $2 \sigma$ below the one that can be derived from the COBE data [6]. The latter is consistent with $b \simeq 1$, which seems not to be in agreement with observed large scale structure, showing an excess of a small scale power.

As it was shown in [7], the partial (10-30\%) replacement of CDM by HDM increases the large scale power and decreases the small scale one compared to pure CDM case. So, such a $\mathrm{CDM}+\mathrm{HDM}$ model, with $b \simeq 1$ and inflationary (flat) spectrum can be made compatible with COBE data. Our situation, however, differs somewhat from the one studied in ref. [7], since there it was assumed only one light neutrino species with a mass $\sim 5-10 \mathrm{eV}$, whereas we end up with 8 times larger concentration and a mass $\sim 1 \mathrm{eV}$. It would follow that in our case one predicts the power spectrum to be more flat than that in [7 for the same percentage of HDM. In any case this issue deserves further consideration.

\section{The Model}

Although most of our analysis was to a large extent model independent, for the sake of illustration we present a simple model based on $G=U(1)_{e} \times U(1)_{\mu} \times U(1)_{\tau} \times U(1)_{n}$. The model is a straightforward extension of that of $\mathrm{BH}$ [15], which is based on the lepton flavor symmetry in the Zee model [27]. On top of $S_{a b}$ fields, one introduces a set of $S U(2)_{L}$ singlet charged scalars $h_{a b}^{-}(a \neq b)$ transforming as $S_{a b}$ under $G$, which have the following couplings to leptons

$$
\Delta L_{Y}=f_{i j} l_{i}^{T} C i \tau_{2} l_{j} h_{i j}^{*}+f_{i n}\left(n^{c}\right)^{T} C e_{i}^{c} h_{i n}+\text { h.c. }
$$

where $e_{i}^{c}$ are the charge conjugates of the right-handed leptons, singlets under $S U(2)_{L}$. Note that here all the fermion fields are left-handed.

The transformation properties of $h_{a b}$ and $S_{a b}$ fields allow for an important additional terms in the scalar potential

$$
\Delta V=\lambda_{a b}\left(\phi_{1}^{T} i \tau_{2} \phi_{2}\right) h_{a b} S_{a b}^{*}+\lambda_{a b c d} h_{a b}^{*} h_{c d} S_{a b} S_{c d}^{*}+h . c .
$$


where the two scalar doublets $\phi_{1}$ and $\phi_{2}$ are necessary because of the antisymmetry of the $\phi \phi h$ coupling [27].

We illustrate here the case $L_{+}=L_{e}-L_{\mu}+L_{\tau}-L_{n^{c}}$, which implies the only non-vanishing VEVs of the $S$ fields to be

$$
<S_{e \mu}>\neq 0 \neq<S_{\mu \tau}>, \quad<S_{\mu n}>\neq 0
$$

The leading radiatively induced neutrino masses are then (see Fig. 1)

$$
\begin{gathered}
m_{\mu \tau} \simeq \frac{1}{16 \pi^{2}}\left(f_{\mu \tau} g_{\tau} \lambda_{\mu \tau}\right) m_{\tau} \frac{<S_{\mu \tau}><H>}{M^{2}} \\
m_{e \mu} \simeq \frac{1}{16 \pi^{2}}\left(f_{e \mu} g_{\mu} \lambda_{e \mu}\right) m_{\mu} \frac{<S_{e \mu}><H>}{M^{2}} \\
m_{e n} \simeq \frac{1}{16 \pi^{2}}\left(f_{e \tau} f_{\tau n} \lambda_{e \tau \tau n}\right) m_{\tau} \frac{<S_{e \mu}><S_{\mu n}>}{M^{2}} \\
m_{\tau n} \simeq \frac{1}{16 \pi^{2}}\left(f_{\tau \mu} f_{\mu n} \lambda_{\tau \mu \mu n}\right) m_{\mu} \frac{<S_{\tau \mu}><S_{\mu n}>}{M^{2}}
\end{gathered}
$$

where $H$ is a linear combination of $\phi_{1}$ and $\phi_{2}$, chosen so as to have $<H>\neq 0$ and $g_{i}$ $(i=e, \mu, \tau)$ are the Yukawa couplings of the orthogonal combination $\phi^{\prime}$ with a vanishing VEV. For simplicity we assume all the scalar masses to be the same $(M)$ Ð. Recall that the above pattern of VEVs can always be achieved in the absence of additional symmetries.

Notice that for $M \simeq M_{W},<S_{\mu \tau}>\simeq M_{W}$, with the phenomenological limit $f_{\mu \tau} \leq 10^{-1}$, $m_{\mu \tau}$ is naturally of the order $10 \mathrm{keV}$. Another way of phrasing this is that $M$ and $<S>$ must be close to $M_{W}$ in order to reproduce the $17 \mathrm{keV}$ neutrino. It is easy to deduce an upper limit $\left\langle S>\lesssim M_{\sim}^{<1} \mathrm{TeV}\right.$, which implies that all the new particles in the model can be detectable in the near future. This is the most appealing feature of the these type of models.

If $f_{e \mu} \sim f_{\mu \tau}, \lambda_{e \mu} \sim \lambda_{\mu \tau}$ one would predict $\theta_{S} \sim m_{e \mu} / m_{\mu \tau} \sim m_{\mu} / m_{\tau} \simeq 0.1$. Unfortunately, the predictions are obscured by the complete arbitrariness of the couplings $g_{i}$ of the

\footnotetext{
${ }^{5}$ In any case, the difference in the masses of the scalars can be reabsorbed in the unknown coupling constants.
} 
second doublet. Furthermore, the model as it stands would not lead to the natural flavor conservation in the quark sector [28]. The simplest way out is to have the doublets $\phi_{\alpha}$ to couple to up and down quarks separately. This requires the existence of a discrete symmetry $D$, such as

$$
\phi_{u} \rightarrow-\phi_{u}, \quad u_{R} \rightarrow-u_{R}, \quad S \rightarrow-S
$$

and the rest of the fields invariant. Now obviously both $\phi_{u}$ and $\phi_{d}$ should have non-vanishing VEVs (due to an above symmetry, one cannot rotate one of the VEVs away). The spontaneously symmetry breaking of $D$ through $\left\langle\phi_{u}\right\rangle \neq 0$ leads at first glance to a catastrophic existence of the domain walls. Fortunately, due to an anomaly, these walls can be shown to decay away before dominating the energy density of the universe [29].

With this $D$ symmetry one has

$$
g_{i}=\frac{m_{i}}{<\phi_{d}>}
$$

and so to arrive at a correct value of $\theta_{S}$ one needs $\frac{f_{e \mu}}{f_{\mu \tau}} \frac{\lambda_{e \mu}}{\lambda \mu \tau} \simeq 10$. Similar adjustment is necessary to explain the hierarchy $m_{e n}, m_{\tau n} \ll m_{\mu \tau}$ which may be needed to comply with the cosmological limits on the number of neutrino species. Again, there is enough freedom to accommodate this requirement through the unknown $\lambda_{i j k l}$ couplings.

\section{Discussion}

In short, our model is a natural and straightforward extension of the BH picture of flavons, i.e. Majorons associated with the lepton flavors. In the limit of conserved $L$, the model is basically phenomenologically indistinguishable from that of $\mathrm{BH}$, except for possible cosmological role of $n$. We do not repeat their analysis here, suffice it to mention their central results:

(a) The "flavon" type models incorporate naturally $17 \mathrm{keV}$ neutrino without requiring any new mass scales. 
(b) The most interesting prediction of $\mathrm{BH}$ which also holds here seems to be the potentially observable $\tau \rightarrow e F$ ( $F$ is a flavon) decay: $B R(\tau \rightarrow e F) \simeq 10^{-4}$.

The principal motivation of our work was to attempt to shed some more light on other central issues of neutrino physics, such as the problems of solar and atmospheric neutrinos and the dark matter problem by adding a light sterile neutrino. Of course, as long as the generalized ZKM lepton number stays unbroken, one ends up with two 4-component neutrinos, one $\nu_{17}$ and another $\nu_{e}$ with mass $\underset{\sim}{<} 10 \mathrm{eV}$, and so no oscillations relevant to the SNP and ANP are possible.

Once again we would like to stress the crucial nature of our gravitationally induced breaking of $L$. Besides providing necessary mass splittings of the order of $10^{-6} \mathrm{eV}$ in both heavy $\left(\nu_{17}\right)$ and light $\left(\nu_{e}\right)$ sectors, it also induces a substantial mass of flavons, of the order of $1 \mathrm{keV}$. The requirement of sufficiently fast decay of flavons yields a lower bound on the $\nu_{e}$ mass $m_{\nu_{e}}>(0.1-1) \mathrm{eV}$ which, in turn, implies at least a few percent of dark matter being hot.

Another important feature of our work is that squared mass difference in the heavy neutrino sector is $\Delta m_{\text {heavy }}^{2} \sim 10^{-2}-10^{-3} \mathrm{eV}^{2}$, which together with maximal mixing is in the right range for the solution of the ANP. This, however, can only work if $\nu_{17} \simeq \nu_{\tau}+\nu_{\mu}^{c}$ since than $\nu_{\mu} \rightarrow \nu_{\tau}$ oscillations can do the job [5]. If the $\nu_{17}$ really exists, the ANP can provide the necessary insight into its structure. We would like to emphasize, though, that its existence is by no means crucial for our work. It is true that without the $\nu_{17}$ none of the other issues under consideration require the existence of a light sterile state. It is only when gravitationally induced effects are the source of the splittings of neutrino masses that $n$ is necessary for a simultaneous solution of the SNP and ANP. We can even reverse the logic of our analysis and say that the solution of the ANP in the context of Planck-scale physics tends to suggest the existence of a neutrino in the $10 \mathrm{keV}$ mass range. Of course, its mixing angle with $\nu_{e}$ could easily be two orders of magnitude smaller than $\theta_{S}$.

As was shown in section $3, \Delta m_{\text {light }}^{2}$ lies in the range $10^{-8}-10^{-5} \mathrm{eV}^{2}$. This overlaps 
with the $\Delta m^{2}$ domain of the MSW solution [30 of the SNP. We should stress, however, that the MSW effect is anyway irrelevant for the SNP in our scenario since the mixing angle is practically equal to $45^{\circ}$. This means that we have the short-wavelength vacuum oscillation $\nu_{e} \rightarrow n^{c}$ solution of the SNP since $\Delta m_{\text {light }}^{2} \gg 10^{-10} \mathrm{eV}^{2}$. Therefore one gets an universal suppression factor $\simeq 1 / 2$ for all the solar neutrino experiments. This is in a good agreement with the results of the Kamiokande [2] SAGE [3] and GALLEX [4] but is at variance with the Homestake data [1]. Further experiments are needed to clarify the situation. The oscillation into a sterile state predicts suppressed neutrino signals in the neutral-current channels in the forthcoming Sudbury Neutrino Observatory [31] experiment.

Our discussion up to now was almost exclusively devoted to the choice $L_{+}$of the conserved generalized ZKM symmetry. It is clear that the situation in the case of $L_{-}$is almost identical; some distinct features are listed in the Table 1 (we should mention that all cases with $n$ in the heavy state are in the potential conflict with the SN 1987A constraints [18], but we appeal to new supernovae to resolve this issue). As far as the other choices, $L_{1}, L_{2}$ and $L_{3}$ are concerned, they lead to one heavy and two massless neutrinos (up to tiny gravitational effects inducing $\sim 10^{-6}-10^{-5} \mathrm{eV}$ masses for the latter) and so do not allow for the Majoron decays. Their properties are still listed in the Table 1, since they naturally allow for the so called "just so" oscillation solution of the SNP, with $\Delta m_{\text {light }}^{2} \simeq 10^{-10}-10^{-11} \mathrm{eV}^{2}$ [32]. The natural way out of the Majoron stability for these cases remains a challenge, since we do not wish to pursue an unappealing possibility of fine-tuning the flavon masses to be sufficiently small.

Last but not least we wish to emphasize the relevance of the predicted electron neutrino mass in the range $0.1-10 \mathrm{eV}$. To obtain what appears to be a favored amount of about twenty per cent hot dark matter in the present-day universe, $\nu_{e}$ mass should be approximately 1 $\mathrm{eV}$ which is in the reach of a future direct observation. 


\section{Acknowledgements}

We would like to thank C. Burgess, J. Cline, A. Dolgov, R. Mohapatra, S. Petcov, R. Schaefer, Q. Shafi, A. Smirnov and J. Valle for useful discussions.

\section{References}

[1] R. Davis, in Proc. XXI Int. Cosmic Ray Conf., ed. R.J. Protheroe, vol. 12 (Univ. of Adelaide Press, Adelaide, Australia, 1990), p. 143.

[2] K.S. Hirata et al., Phys. Rev. Lett. 65, 1297 (1990); Phys. Rev. D 44, 2141 (1991).

[3] SAGE Collaboration, A.I. Abazov et al., Phys. Rev. Lett. 67, 3332 (1991).

[4] GALlEX Collaboration, P. Anselmann et al., Phys. Lett. B 285, 376, 390 (1992).

[5] Kamiokande II collaboration, K.S. Hirata et al., Phys. Lett. B 280, 146 (1992); IMB Collaboration, D. Gasper et al., Phys. Rev. Lett. 66, 2561 (1991); E.W. Beier et al., Phys. Lett. B 283, 446 (1992).

[6] G.F. Smoot et al., COBE preprint, 1992 (Ap. J. Lett., in press).

[7] R.K. Schaefer, Q. Shafi, preprint BA-92-28(1992); preprint IC/92/118, BA-92-45 (1992); A. van Dalen, R.K. Schaefer, preprint BA-91-67 (1992).

[8] J.J. Simpson, Phys. Rev. Lett. 54, 1891 (1985); Phys. Lett. 174B, 113 (1986); J.J. Simpson, A. Hime, Phys. Rev. D 39, 1825 (1989); A. Hime, J.J. Simpson, Phys. Rev D 39, 1837 (1989); A. Hime, N.A. Jelley, Phys. Lett. B 257, 441 (1991); B. Sur et al., Phys. Rev. Lett. 66, 2444 (1991); I. Žlimen et al., Phys. Rev. Lett. 67, 560 (1991).

[9] T. Altzitzoglou et al., Phys. Rev. Lett. 55, 799 (1985); T. Ohi et al., Phys. Lett. B 160, 322 (1985); V.M. Datar et al., Nature (London), 318, 547 (1985); A. Apalikov et al., 
Pis'ma Zh. Eksp. Theor. Fiz. 42, 233 (1985) [JETP Lett. 42, 289 (1985)]; J. Markey, F. Boehm, Phys. Rev. C 32, 2215 (1985); D.W. Hetherington et al., Phys. Rev. C 36, 1504 (1987); M.J.G. Borge et al., Phys. Scr. 34, 591 (1986).

[10] See, e.g. S.L. Glashow, Phys. Lett. B 256, 218 (1991); L. Bento, J.W.F. Valle, Phys. Lett. B 264, 373 (1991); K.S. Babu, R.N. Mohapatra, Phys. Rev. Lett. 67, 1498 (1991); K. Choi, A. Santamaria, Phys. Lett. B 267, 504 (1991); D. Choudhury, U. Sarkar, Phys. Lett. B 268, 96 (1991); G.K. Leontaris, C.E. Vayonakis, J.D. Vergados, Ioannina preprint IOA- 269, 1992; K.S. Babu R.N. Mohapatra, I.Z. Rothstein, Phys. Rev. D 45, R5 (1992). In the last paper a comprehensive list of literature on the subject can be found.

[11] D.O. Caldwell, P. Langacker, Phys. Rev. D 44, 823 (1991); A.Yu. Smirnov, talk at the EPS Conference, CERN, July 1991 (unpublished); A.Yu. Smirnov, J.W.F. Valle, preprint FTUV/91-38, 1991; J. Peltoniemi, A.Yu. Smirnov, J.W.F. Valle, preprint FTUV/92-6, 1992; K.S. Babu, R.N. Mohapatra, I.Z. Rothstein, Phys. Rev D 45, R5 (1992); K.S. Babu, R.N. Mohapatra, preprint UMDHEP 92-150, 1992; E. Ma, Phys. Rev. Lett. 68, 1981 (1992); C.P Burgess, J.M. Cline, M.A. Luty, Phys. Rev. D 46, 364 (1992).

[12] Ya.B. Zeldovich, Dokl. Akad. Nauk SSSR 86, 505 (1952); E.J. Konopinski, H. Mahmoud, Phys. Rev. 92, 1045 (1953).

[13] M.J. Dugan, G. Gelmini, H. Georgi, L.J. Hall, Phys. Rev. Lett. 54, 2302 (1985); J.W.F. Valle, Phys. Lett. B 159, 49 (1985). This lepton charge was first introduced in another context by S.T. Petcov, Phys. Lett. 110B, 245 (1982).

[14] ALEPH Collaboration, D. Decamp et al., Phys. Lett. B 235, 399 (1990); OPAL Collaboration, M.Z. Akrawy et al., Phys. Lett. B 240, 497 (1990); DELPHI Collaboration, P. Abreu et al., Phys. Lett. B 241, 435 (1990); L3 Collaboration, B. Adeva et al., Phys. Lett. B 249, 341 (1990). 
[15] R. Barbieri, L. Hall, Nucl. Phys. B364, 27 (1991); Z. Berezhiani, (unpublished).

[16] R. Barbieri, J. Ellis, M.K. Gaillard, Phys. Lett. 90B, 249 (1980); E. Akhmedov, Z. Berezhiani, G. Senjanović, preprint IC/92/79, SISSA-83/92/EP, LMU-04/92.

[17] D. Grasso, M. Lusignoli, M. Roncadelli, Rome preprint n.868, FNT/T 92/07, 1992.

[18] G. Raffelt, D. Seckel, Phys. Rev. Lett. 60, 1793 (1988); K.J.F. Gaemers, R. Gandhi, J.M. Lattimer, Phys. Rev D 40, 309 (1989); J.A. Grifols, E. Massó, Phys. Lett. B 242, 77 (1990); R. Gandhi, A. Burrows, Phys. Lett. B 246, 149 (1990); B 261, 519(E) (1991); M.S. Turner, Phys. Rev. D 45, 1066 (1992); S. Dodelson, J.A. Frieman, M.S. Turner, Phys. Rev. Lett. 68, 2572 (1992); A. Burrows, R. Gandhi, M.S. Turner, Phys. Rev. Lett. 68, 3834 (1992).

[19] See, e.g. A.Yu. Smirnov and J.W.F. Valle, ref. [4]. The phenomenology of the system with the conserved lepton charge $L_{+}$(with $n$ being an active neutrino of the fourth generation) was analysed in a model-independent way by M. Lusignoli, Phys. Lett. 168B, 307 (1985).

[20] A.D. Dolgov, Yad. Fiz. 33, 1309 (1981) [Sov. J. Nucl. Phys. 33, 700 (1981)]; M.Yu. Khlopov, S.T. Petcov, Phys. Lett. B 99, 117 (1981); B 100, 520 (1981) (E); A.D. Dolgov, D.P. Kirrilova, Intern. J. Mod. Phys. A 3, 267 (1988).

[21] R. Barbieri, A. Dolgov, Phys. Lett. B 237, 440 (1990); Nucl. Phys. B349, 743 (1991); K. Kainulainen, Phys. Lett. B 244, 191 (1990); K. Enqvist, K. Kainulainen, J. Maalampi, Nucl. Phys. B349, 754 (1990).

[22] K. Enqvist, K. Kainulainen, M. Thomson, Phys. Rev. Lett. 68, 744 (1992); Nucl. Phys. B373, 498 (1992).

[23] K.A. Olive, D.N. Schramm, G. Steigman, T.P. Walker, Phys. Lett. B 236, 454 (1990). 
[24] J.F.Wilkerson et al, in Proceedings of the 14th International Conference on Neutrino Physics and Astrophysics, CERN, Geneva, June 10-15, 1990 [Nucl.Phys.B Proc.Suppl. 19, 215, 1991].

[25] A. Hime, R.J.N. Phillips, G.G. Ross, S. Sarkar, Phys. Lett. B 260, 381 (1991).

[26] E.Kh. Akhmedov, Z.G. Berezhiani, R.N. Mohapatra and G. Senjanović, in preparation.

[27] A. Zee, Phys. Lett. 93B, 389 (1980); 161B, 141 (1985).

[28] S.L. Glashow, S. Weinberg, Phys. Rev. D 15, 1958 (1977); E.A. Paschos, Phys. Rev. D 15, 1966 (1977).

[29] J. Preskill, S.T. Trivedi, F. Wilczek, M.B. Wise, Nucl. Phys. B363, 207 (1991).

[30] S.P. Mikheyev, A.Yu. Smirnov, Yad Fiz 42, 1441 (1985) [Sov. J. Nucl. Phys. 42, 913 (1985)]; L. Wolfenstein, Phys. Rev. D 17, 2369 (1978).

[31] SNO Collaboration, G. Aardsma et al., Phys. Lett. B 194, 321 (1987).

[32] S.M. Bilenky, B. Pontecorvo, Phys. Rep. 41, 225 (1978); V. Barger, R.J.N. Phillips, K. Whisnant, Phys. Rev. D 24, 538 (1981); Phys. Rev. D 43, 1110 (1991); S.L. Glashow, L.M. Krauss, Phys.Lett. B 190, 199 (1987); A. Acker, S. Pakvasa, J. Pantaleone, Phys. Rev. D 43, 1754 (1991); P.I. Krastev, S.T. Petcov, preprint CERN-TH. 6401/92 (1992). 


\begin{tabular}{c|c|c|c|c|c}
\hline $\begin{array}{c}\text { Generalized } Z K M \\
\text { lepton number }\end{array}$ & $\begin{array}{c}\text { Content of heavy } \\
\text { neutrino" } \nu_{17} "\end{array}$ & ANP & SNP & $N_{\nu}$ & HDM \\
\hline$L_{+}\left(\theta_{S} \simeq \theta_{e \tau}\right)$ & $\nu_{\tau}+\nu_{\mu}^{c}$ & $\nu_{\mu} \leftrightarrow \nu_{\tau}$ & $\nu_{e} \leftrightarrow n^{c}(S W)$ & $3-4$ & $\nu_{e}$ \\
& $\nu_{\tau}+n$ & - & $\nu_{e} \leftrightarrow \nu_{\mu}(S W)$ & 4 & $\nu_{e}$ \\
\hline$L_{-}\left(\theta_{S} \simeq \theta_{e n^{c}}\right)$ & $n^{c}+\nu_{\mu}^{c}$ & $\nu_{\mu} \leftrightarrow n^{c}$ & $\nu_{e} \leftrightarrow \nu_{\tau}(S W)$ & 4 & $\nu_{e}$ \\
\hline$L_{1}\left(\theta_{S} \simeq \theta_{e \tau}\right)$ & $n^{c}+\nu_{\tau}^{c}$ & - & $\nu_{e} \leftrightarrow \nu_{\mu}(V A)$ & 4 & $\nu_{e}$ \\
\hline$\left.\nu_{S} \simeq \theta_{e n^{c}}\right)$ & $\nu_{\tau}+\nu_{\mu}^{c}$ & $\nu_{\mu} \leftrightarrow \nu_{\tau}$ & $\nu_{e} \leftrightarrow n^{c}(J S)$ & 3 & $?$ \\
\hline$L_{3}\left(\theta_{S} \simeq \theta_{e n^{c}} \simeq \theta_{e \tau}\right)$ & $n^{c}+\nu_{\mu}^{c}$ & $\nu_{\mu} \leftrightarrow n^{c}$ & $\nu_{e} \leftrightarrow \nu_{\tau}(J S)$ & 4 & $?$ \\
\hline
\end{tabular}

Table 1. Summary of heavy neutrino composition and solutions for the SNP and ANP for generalized lepton charges $L_{+}, L_{-}$and $L_{1,2,3} . S W$ and $J S$ stand for the solutions of the SNP through short wavelength (averaged) vacuum oscillations $\left(\Delta m^{2} \simeq 10^{-8}-10^{-5} \mathrm{eV}^{2}\right)$ and "just so" oscillations $\left(\Delta m^{2} \simeq 10^{-10}-10^{-11} \mathrm{eV}^{2}\right)$ respectively. Also shown are the effective number of neutrino species at the time of nucleosynthesis $N_{\nu}$ and the composition of HDM. Question marks indicate the problem with the decay of massive flavons explained in section 5 . 


\section{Figure caption}

Fig. 1. One-loop diagrams which induce the neutrino mass terms $m_{i j}$ (a) and $m_{i n}(\mathrm{~b}) ; i, j$ take the values allowed by the $L$ symmetry. $H$ and $\phi^{\prime}$ are the linear combinations of $\phi_{1}$ and $\phi_{2}$ with non-vanishing and vanishing VEVs respectively. 